\title{
Bouveret syndrome: a tough nut to crack
}

Ijlal Akbar Ali, MD, ${ }^{1}$ Sultan Mahmood, $\mathbf{M D},{ }^{2}$ William M. Tierney, MD $^{1}$

A 48-year-old morbidly obese woman presented to the emergency department with a 3-day history of severe right upper-quadrant abdominal pain associated with nausea and vomiting. She did not describe any fevers or chills. The patient had a history of biliary colic for the previous 3 to 4 years and has been told she had cholelithiasis.

A CT scan showed a large $3-\mathrm{cm}$ gallstone in the duodenum with a fistulous connection to the gallbladder (Fig. 1). The gastroenterology service was consulted for possible endoscopic stone extraction.

Endoscopic examination (Video 1, available online at www.VideoGIE.org) found a large gallstone impacted in the duodenal bulb with a fistulous opening identified at the 5 o'clock position (Fig. 2A). A 12-mm ERCP stone extraction balloon catheter was passed beyond the stone, and contrast dye was injected to define the anatomy of the duodenum. The balloon was inflated to $12 \mathrm{~mm}$, and attempts were made to pull the stone in a retrograde fashion through the pylorus into the stomach. However, after multiple attempts, we were unsuccessful in pulling the stone through the pylorus because of its size. Next, a 3- $\times$ 6-cm Roth net (US Endoscopy, Mentor, Ohio, USA) was used to capture the stone, but it was too large to be completely captured in the net. Attempts to withdraw the stone into the stomach resulted in the stone slipping out of the net. A large snare was also unable to fully engage the large stone.

We then proceeded with sterile saline solution infusion into the duodenum and electrohydraulic lithotripsy (Autolith; Northgate Technologies, Elgin, Ill, USA) at initial settings of $50 \mathrm{~W}, 5$ pulses per activation. After initial limited fracture of the stone, the settings were increased to $70 \mathrm{~W}, 5$ pulses per activation. This was a timeconsuming process that eventually successfully fractured the stone into several large fragments (Fig. 2B). These pieces were then pulled to the gastric body with a snare. All stone fragments from the stomach were removed with the Roth net. The fistulous connection was identified and inspected more closely (Fig. 2C). The second portion of the duodenum was examined to ensure no stone fragments remained. Finally, injection of contrast material after balloon occlusion of the duodenal bulb showed no extravasation of contrast material through the fistula and no distal filling defects in the duodenum. The patient did well after the procedure; on postdischarge follow-up with the surgery service, it was decided to defer cholecystectomy and fistula repair and to monitor for any recurrent symptoms caused by significant comorbidities (morbid obesity with basal metabolic index of $56 \mathrm{~kg} / \mathrm{m}^{2}$ ).

Bouveret syndrome is an uncommon and very rare form of gallstone ileus caused by the passage and impaction of large gallstones through a cholecystoduodenal fistula into the duodenum, resulting in gastric outlet obstruction. ${ }^{1}$ CT or magnetic resonance imaging with contrast material may demonstrate the fistula, and when oral contrast material is given, there may be evidence of extravasation of contrast material into the gallbladder. Open surgical therapy is associated with high morbidity and mortality. Endoscopic removal is the preferred initial approach, and although previous reports suggest success rates of only $10 \%$, more advanced endoluminal lithotripsy can be associated with a much higher rate of complete stone clearance. ${ }^{2}$

Various endoscopic modalities have been used in the successful management of Bouveret syndrome. Electrohydraulic lithotripsy is a potential option. It is commonly used to fragment pancreatic and biliary stones and is readily available in many hospitals. A double-channel therapeutic gastroscope is typically used to allow for continuous irrigation to minimize the risk of damage, perforation, and bleeding by shock wave dispersion.

It is imperative to remove all stone fragments to prevent gallstone ileus. ${ }^{2}$ Although closure of the fistula is debatable, it is often considered unnecessary because of spontaneous closure, especially when the cystic duct is patent and no

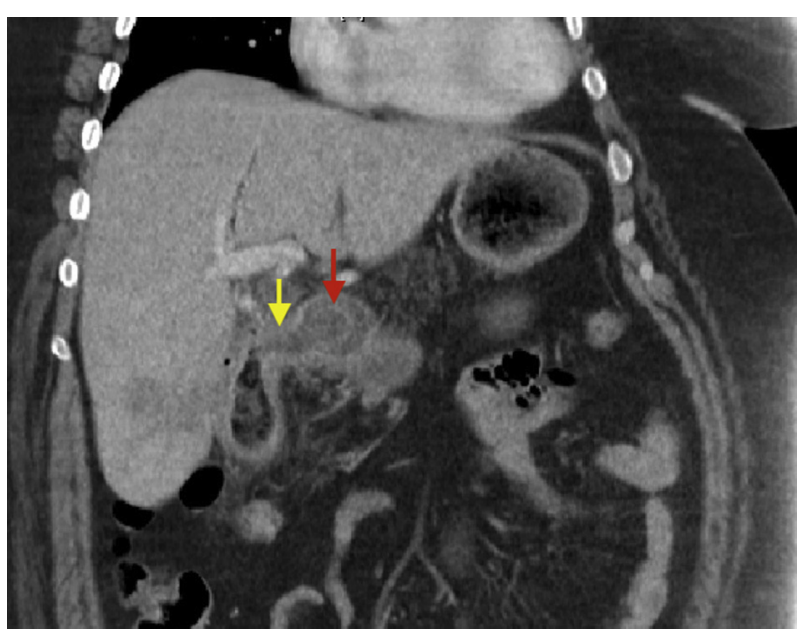

Figure 1. CT scan demonstrating choledochoduodenal fistula (yellow arrow) and the large $3-\mathrm{cm}$ gallstone (red arrow). 

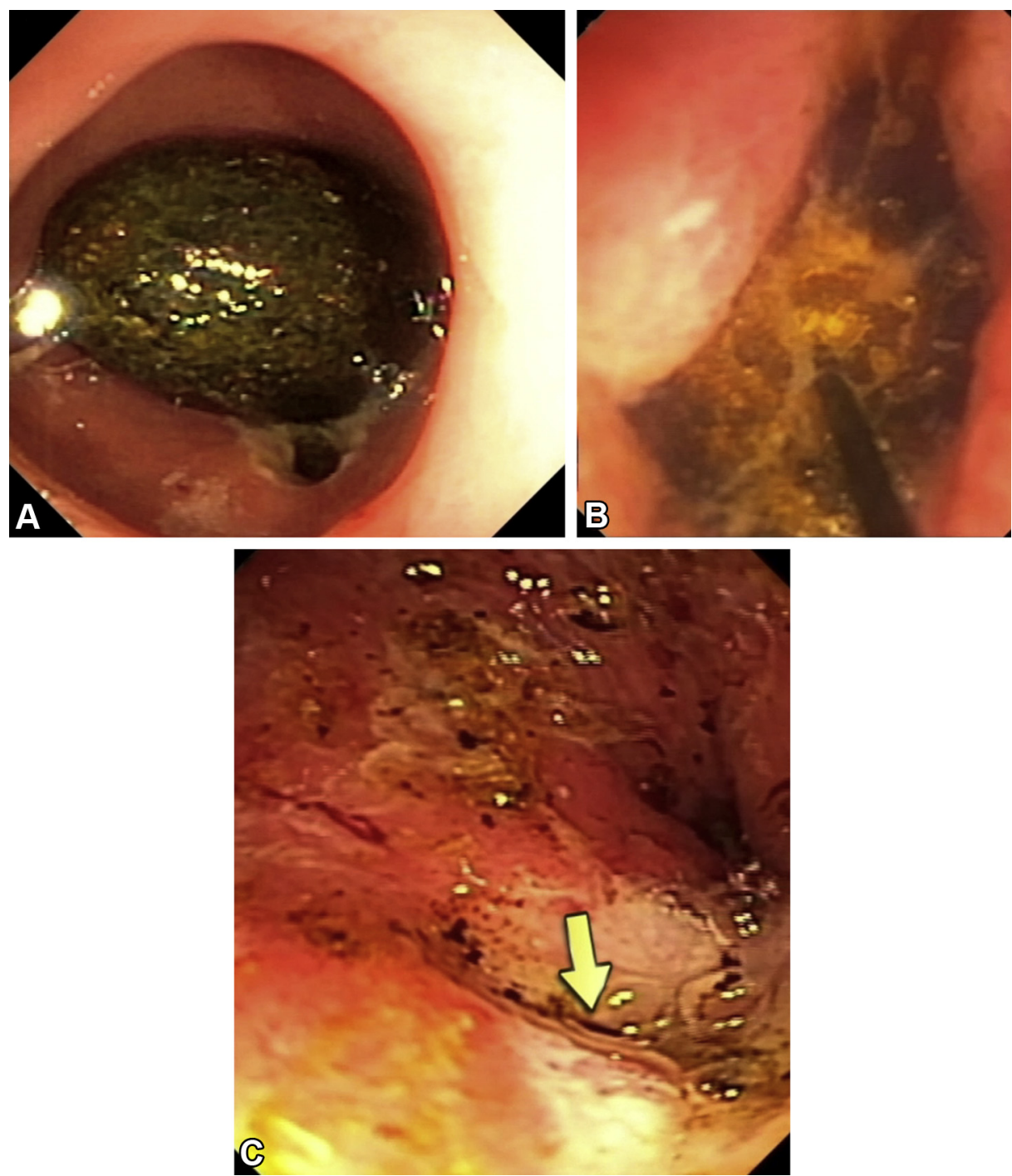

Figure 2. Successful endoscopic removal of gallstone. A, Gallstone impacted in duodenal bulb with fistula at 5'o clock position. B, Electrohydrolithotripsy probe used to fracture stone. C, Fistulous connection demonstrating (yellow arrow) after complete removal of stone.

residual stones are present, as was the case in our patient. Proponents of fistula repair argue that there is an increased risk of recurrence and increased risk of gallbladder cancer. Surgical closure can, therefore, be considered at the time of cholecystectomy, especially in young and healthy patients. ${ }^{3}$

\section{DISCLOSURE}

All authors disclosed no financial relationships relevant to this publication.

\section{REFERENCES}

1. Cappell MS, Davis M. Characterization of Bouveret's syndrome: a comprehensive review of 128 cases. Am J Gastroenterol 2006;101:2139-46.
2. Lowe AS, Stephenson S, Kay $C L$, et al. Duodenal obstruction by gallstones (Bouveret's syndrome): a review of the literature. Endoscopy 2005;37:82-7.

3. Caldwell KM, Lee SJ, Leggett PL, et al. Bouveret syndrome: current management strategies. Clin Exp Gastroenterol 2018;11:69-75.

Department of Digestive Diseases and Nutrition, Oklahoma University Health Sciences Center, Oklahoma City, Oklahoma, USA (1), Marshfield Clinic Gastroenterology, Marshfield, Wisconsin, USA (2).

Copyright (c) 2018 American Society for Gastrointestinal Endoscopy. Published by Elsevier Inc. This is an open access article under the CC BYNC-ND license (http://creativecommons.org/licenses/by-nc-nd/4.0/).

https://doi.org/10.1016/j.vgie.2018.11.007 